\title{
Physical activity and sedentary time among preschoolers in centre-based childcare: a systematic review
}

\author{
Kathleen T. O'Brien ${ }^{1}$, Leigh M. Vanderloo², Brianne A. Bruijns ${ }^{3}$, Stephanie Truelove ${ }^{3}$ and Patricia Tucker ${ }^{4 *}$
}

\begin{abstract}
Background: Many preschoolers spend a substantial portion of their day enrolled in centre-based childcare; the amounts of physical activity and sedentary time accumulated in this environment are critical to preschoolers' ability to meet movement guidelines. The purpose of this systematic review was to provide a comprehensive overview of the objectively assessed physical activity and sedentary time of preschoolers in centre-based childcare (registration no. CRD42016033502).

Methods: Eight online databases were searched using terms related to physical activity, sedentary time, preschoolers and centre-based childcare. Published, peer-reviewed primary studies written in English that objectively assessed (via accelerometry) the physical activity and sedentary time of preschoolers (2-5 years) in centre-based childcare were included.

Results: Fifty-five studies (published 2004-2017) from 11 countries, representing 13,956 participants were included. Studies reported light physical activity $(n=38)$ ranging from 2.94 to 29.96 mins/hr, moderate-to-vigorous physical activity ( $n=46)$ which ranged from 1.29 to $22.66 \mathrm{mins} / \mathrm{hr}$, and total physical activity $(n=42)$ ranging from 4.23 to 47.17 mins/hr. Sedentary time ( $n=47)$ ranged from 12.38 to $55.77 \mathrm{mins} / \mathrm{hr}$.

Conclusion: Physical activity and sedentary time were highly varied and inconsistent between studies; therefore, it is difficult to determine preschoolers' true amount of physical activity and sedentary time during childcare hours. Despite this variability, preschoolers were noted to participate in high rates of sedentary time in this setting. The lack of homogeneity is an important finding in and of itself as it highlights the lack of consistency in measuring, processing, and reporting paediatric physical activity data.
\end{abstract}

Keywords: preschoolers, physical activity, sedentary time, centre-based childcare, systematic review, accelerometry

\section{Introduction}

Levels of physical activity and sedentary time among young children have been widely examined and discussed in the literature [1-4]. To understand the degree to which this population's activity levels are associated with health outcomes, many countries have established physical activity and sedentary behaviour guidelines for young children under 5 years [5-8]. Specific to Canada, the 24-Hour Movement Guidelines recommend participation in at least 180 minutes

\footnotetext{
* Correspondence: ttucker2@uwo.ca

${ }^{4}$ School of Occupational Therapy, Faculty of Health Sciences, University of Western Ontario, 1201 Western Road, Elborn College, Room 2547, London N6G 1H1, Ontario, Canada

Full list of author information is available at the end of the article
}

of physical activity per day for children aged 1-4, including 60 minutes of energetic play (moderate-to vigorous-physical activity [MVPA]) for those 3-4 years [7]. In Canada, Australia, and New Zealand screen-viewing should not exceed more than $60 \mathrm{~min}$ for children 2-4 years, and those under 2 should not engage in any screen use [7]. At 5 years of age, children should engage in 60 minutes of MVPA each day, and limit recreational screen-viewing to $120 \mathrm{~min}$ per day [8]. These guidelines provide benchmarks for parents, public health representatives, and early childhood educators to strive to provide opportunities and support for young children to meet these recommendations. 
In an effort to improve the activity levels of young children, the childcare environment may be a worthwhile setting to intervene - many children are enrolled in these programs and spend a large proportion of their time therein $[9,10]$. In examining this environment, a large Canadian-based study of preschoolers $(n=297)$ found that in comparison to those attending full-day kindergarten and home-based childcare, young children enrolled in centre-based care spent the most time being sedentary at $41.62 \mathrm{mins} / \mathrm{hr}$ [2]. Similarly, Vanderloo et al. (2014) reported in their Canadian study that preschoolers in centre-based childcare $(n=71)$ only spent $1.58 \mathrm{mins} / \mathrm{hr}$ in MVPA [11]. In contrast, Mazzucca et al. (2017) reported that children engaged in 55 minutes of MVPA per childcare day, and that physical activity levels varied between indoor and outdoor activities - a substantial variation from the results produced by Vanderloo and colleagues [12]. Young children have been reported to spend a considerable amount of time in childcare, [10] and in these venues, the rates of physical activity have been documented to be low and sedentary time high; therefore, centre-based childcare represents an ideal setting to foster participation in the recommended amount of physical activity, while undertaking efforts to discourage excessive sedentary time [13-17].

A systematic review of objectively measured physical activity and sedentary time (accelerometers only - the gold standard for this population [18]) of preschoolers' waking hours has been conducted [3]. Specifically, Hnatiuk et al. (2014) reported that the proportion of time spent in these behaviours varied greatly, ranging from 2 to $41 \%$ for MVPA and from 34 to $94 \%$ for sedentary time, as a result of differences in study design and methods of data processing [3]. Consequently, a clearer picture of young children's activity behaviours is needed, specifically in environments like childcare, where centre characteristics have been noted to be a strong influence on these behaviours [1, 19]. While a recent review by Vanderloo, Martyniuk, and Tucker (2015) explored both physical activity and sedentary time of preschoolers in home-based childcare facilities, [20] no systematic review has looked at objectively measured physical activity and sedentary time of preschoolers in centre-based childcare. With research pertaining to the centre-based childcare environment rapidly arising in physical activity literature, as well as recent improvements in activity measurement protocols, it is timely to undertake a synthesis of this work to direct future research efforts and interventions in this setting, as well as government policy. Therefore, the purpose of this study was to systematically review preschoolers' physical activity and sedentary time during centre-based childcare hours, as measured by accelerometry.

\section{Methods}

This review was registered with PROSPERO (no. CRD42016033502), and adheres to the PRIMSA statement for systematic reviews [21, 22].

\section{Search Strategy}

In consultation with a Health Sciences Librarian, a comprehensive search strategy was developed and used to explore young children's physical activity and sedentary time during centre-based childcare hours. Eight electronic databases were searched: CINAHL, Medline, ProQuest, PsychInfo, EMBASE, Scopus, Sport Discus, and Physical Education Index. Search terms focused on physical activity, sedentary time, preschoolers, and centre-based childcare (see Table 1 for a sample search strategy). Database searches ceased on February 10, 2017. Manual searches of four journals' (i.e., Pediatric Exercise Science, Medicine and Science in Sports and Exercise, Journal of Physical Activity and Health, and International Journal of Behavioral Nutrition and Physical Activity) "in press" or "ahead of print" sections, as well as the reference lists of included studies, were reviewed to ensure a thorough and comprehensive search was undertaken. International experts in the field of interest were also contacted to ensure all appropriate literature was captured. The search results were exported and saved in Mendeley (version 1.17.9; referencing software), where duplicates were manually deleted to establish a complete list of articles for screening.

\section{Study Eligibility}

Study eligibility criteria included: 1) primary studies; 2) written in English; 3) published in a peer-reviewed journal; 4) healthy (i.e., free from chronic diseases or developmental delays) preschool children (2 to 5 years) enrolled in centre-based childcare; 5) physical activity and/or sedentary time during centre-based childcare hours measured via accelerometry; and, 6) physical activity and/or sedentary time measured for at least 3 hours on one or more days.

\section{Screening for Inclusion}

The titles and abstracts of all studies captured from the database searches were reviewed independently by two researchers. To ensure that all eligibility criteria were considered, reviewers used a screening form developed by the research team, and adapted from previous studies $[16,20]$. The reviewers discussed conflicting views on the eligibility of an article, and a third researcher was consulted when necessary. All articles that were deemed eligible for inclusion were subsequently reviewed in their full-text form. In instances where the full-text article could not be retrieved via the university's library repository, authors were emailed directly by the research team. 
Table 1 Sample Search Strategy (EMBASE)

\begin{tabular}{|c|c|c|c|}
\hline$\#$ & Search Term & Results & Search Type \\
\hline 1 & preschool child/ & 547180 & Advanced \\
\hline 2 & "preschoolers".mp & 5836 & Advanced \\
\hline 3 & toddler.mp. & 7394 & Advanced \\
\hline 4 & toddlers.mp. & 7518 & Advanced \\
\hline 5 & "early years".mp. & 4177 & Advanced \\
\hline 6 & "early childhood".mp. & 29039 & Advanced \\
\hline 7 & "preschool-aged child".mp. & 47 & Advanced \\
\hline 8 & "preschool-aged children".mp. & 1616 & Advanced \\
\hline 9 & "young child".mp. & 4684 & Advanced \\
\hline 10 & "young children".mp. & 51507 & Advanced \\
\hline 11 & $\begin{array}{l}1 \text { OR } 2 \text { OR } 3 \text { OR } 4 \text { OR } 5 \text { OR } 6 \\
\text { OR } 7 \text { OR } 8 \text { OR } 9 \text { OR } 10\end{array}$ & 1459133 & Advanced \\
\hline 12 & childcare.mp OR exp child care/ & 64155 & Advanced \\
\hline 13 & "childcare centre".mp. & 31 & Advanced \\
\hline 14 & "centre based childcare".mp. & 17 & Advanced \\
\hline 15 & "center based childcare".mp. & 18 & Advanced \\
\hline 16 & "center-based childcare".mp. & 18 & Advanced \\
\hline 17 & "centre-based childcare"mp. & 17 & Advanced \\
\hline 18 & "day care".mp. & 14895 & Advanced \\
\hline 19 & "early learning centre".mp. & 1 & Advanced \\
\hline 20 & "early learning center".mp. & 2 & Advanced \\
\hline 21 & physical activity.mp. OR physical activity/ & 155492 & Advanced \\
\hline 22 & exercise.mp. & 433622 & Advanced \\
\hline 23 & movement.mp. & 361251 & Advanced \\
\hline 24 & "active play".mp. & 220 & Advanced \\
\hline 25 & "locomotor activity".mp. & 24357 & Advanced \\
\hline 26 & "motor activity".mp. & 54511 & Advanced \\
\hline 27 & "physical exertion".mp. & 2798 & Advanced \\
\hline 28 & "active movement".mp. & 1274 & Advanced \\
\hline 29 & "outdoor play".mp. & 217 & Advanced \\
\hline 30 & outdoor time.mp. & 250 & Advanced \\
\hline 31 & recess.mp. & 115 & Advanced \\
\hline 32 & "sedentary behaviour".mp. & 1547 & Advanced \\
\hline 33 & sedentary lifestyle/ or sedentary.mp & 34250 & Advanced \\
\hline 34 & Inactive.mp. & 106149 & Advanced \\
\hline 35 & stationary.mp. & 57673 & Advanced \\
\hline 36 & "physical inactivity".mp. & 9087 & Advanced \\
\hline 37 & "sedentary activity".mp. & 597 & Advanced \\
\hline 38 & $\begin{array}{l}12 \text { OR } 13 \text { OR } 14 \text { OR } 15 \text { OR } 16 \\
\text { OR } 17 \text { OR } 18 \text { OR } 19 \text { OR } 20\end{array}$ & 170150 & Advanced \\
\hline 39 & $\begin{array}{l}21 \text { OR } 22 \text { OR } 23 \text { OR } 23 \text { OR } 24 \text { OR } 25 \\
\text { OR } 26 \text { OR } 27 \text { OR } 28 \text { OR } 29 \text { OR } 30 \text { OR } \\
31 \text { OR } 32 \text { OR } 33 \text { OR } 34 \text { OR } 35 \text { OR } \\
36 \text { OR } 37\end{array}$ & 1593829 & Advanced \\
\hline 40 & 11 AND 38 AND 39 & 802 & Advanced \\
\hline
\end{tabular}

Note: This table was originally published in a review by Truelove et al. [51] and has been reproduced here
Three researchers independently read each article in its entirety, utilizing a full-text screening form designed in advance. Discrepancies in decisions to include or exclude, as well as any concerns regarding eligibility were directed to a fourth researcher.

\section{Extraction of Data}

After the final list of included articles was established, all relevant data were extracted. The information collected via the extraction table included authors, the year of publication, the country in which the study was conducted, information regarding the sample (e.g., size, age range), the model of accelerometer used (e.g., Actical, ActiGraph, etc.), data processing decisions (e.g., wear time, epoch length, cut-points applied, etc.) and levels of physical activity (light, MVPA, total) and sedentary time reported (e.g., $\operatorname{mins} / \mathrm{hr}, \%$ of time, etc.).

\section{Quality Assessment}

The quality of the studies was assessed using the checklist proposed by Downs and Black [23]. All articles were assessed by two reviewers, with a third reviewer serving as an arbitrator, if necessary. For randomized controlled trials, the full checklist (27 questions) was used. A modified version of the checklist (10 questions) was applied to all other study types, and is in-line with previous research studies [24, 25]. The quality score of each article can be found in Table 2. Although not established a priori, all studies included were of high quality (i.e., scored from 21-30 using the full checklist, or 7-10 using the modified checklist) $[23,24]$.

\section{Data Synthesis and Analysis}

To facilitate data synthesis, all included studies were separated into distinct categories depending on the model of accelerometer used. In all instances, the mean hourly rate of physical activity (light physical activity [LPA], MVPA, total physical activity [TPA]) and sedentary time was used to allow for easy comparison between studies. If data for particular intensity levels were not provided, simple calculations were carried out on the basis of available data in the paper. For example, if TPA was not reported, authors summed time spent in LPA and MVPA to derive this final number. For each accelerometer model, the mean for each intensity level across studies was calculated. Data for each level of activity intensity were grouped and synthesized into ranges. Meta-analyses were unable to be conducted given the heterogeneity of the reported study outcomes.

\section{Results}

Database Searches

After searching eight electronic databases, 10,542 articles were captured and uploaded into Mendeley. An additional 


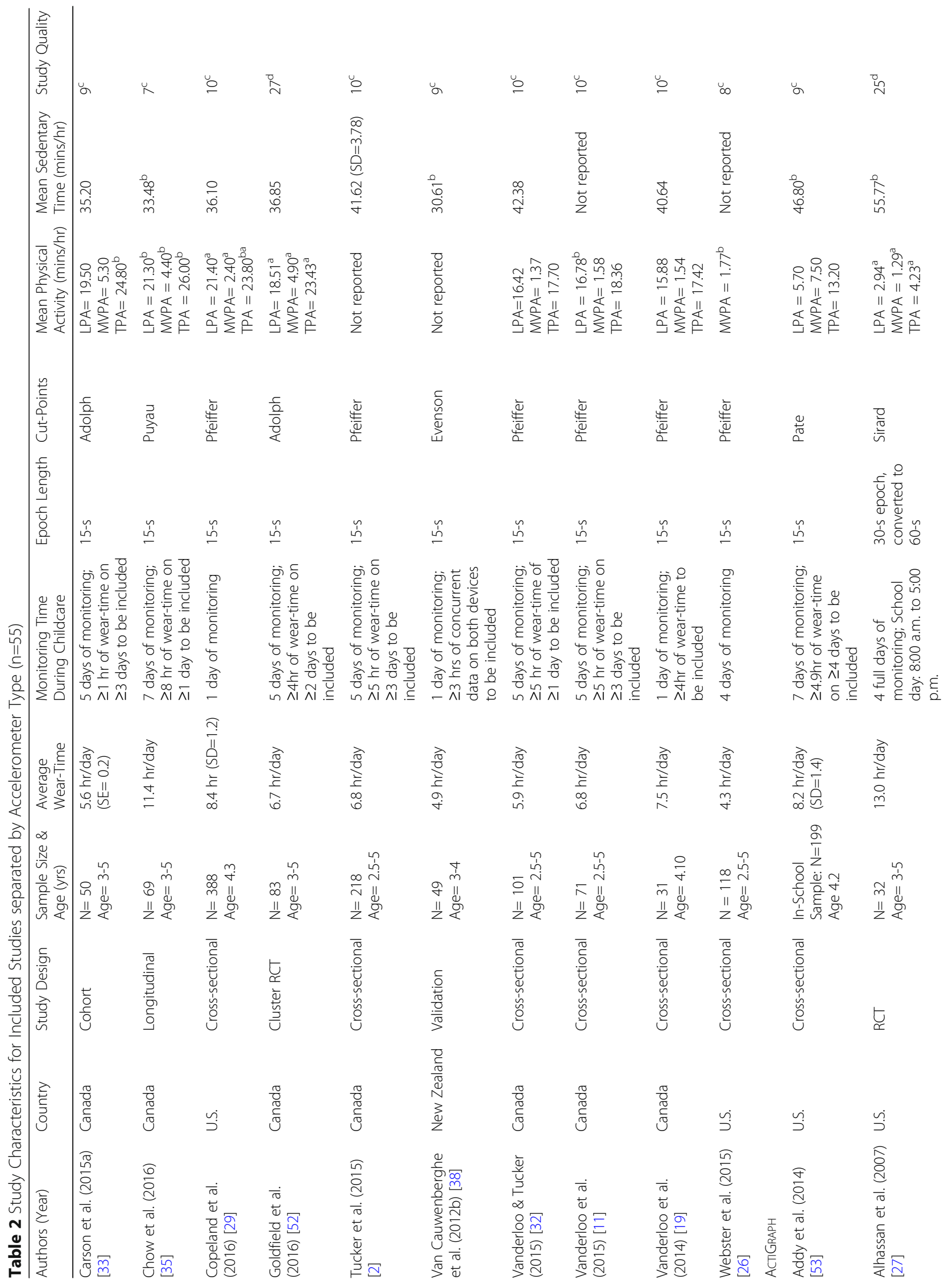




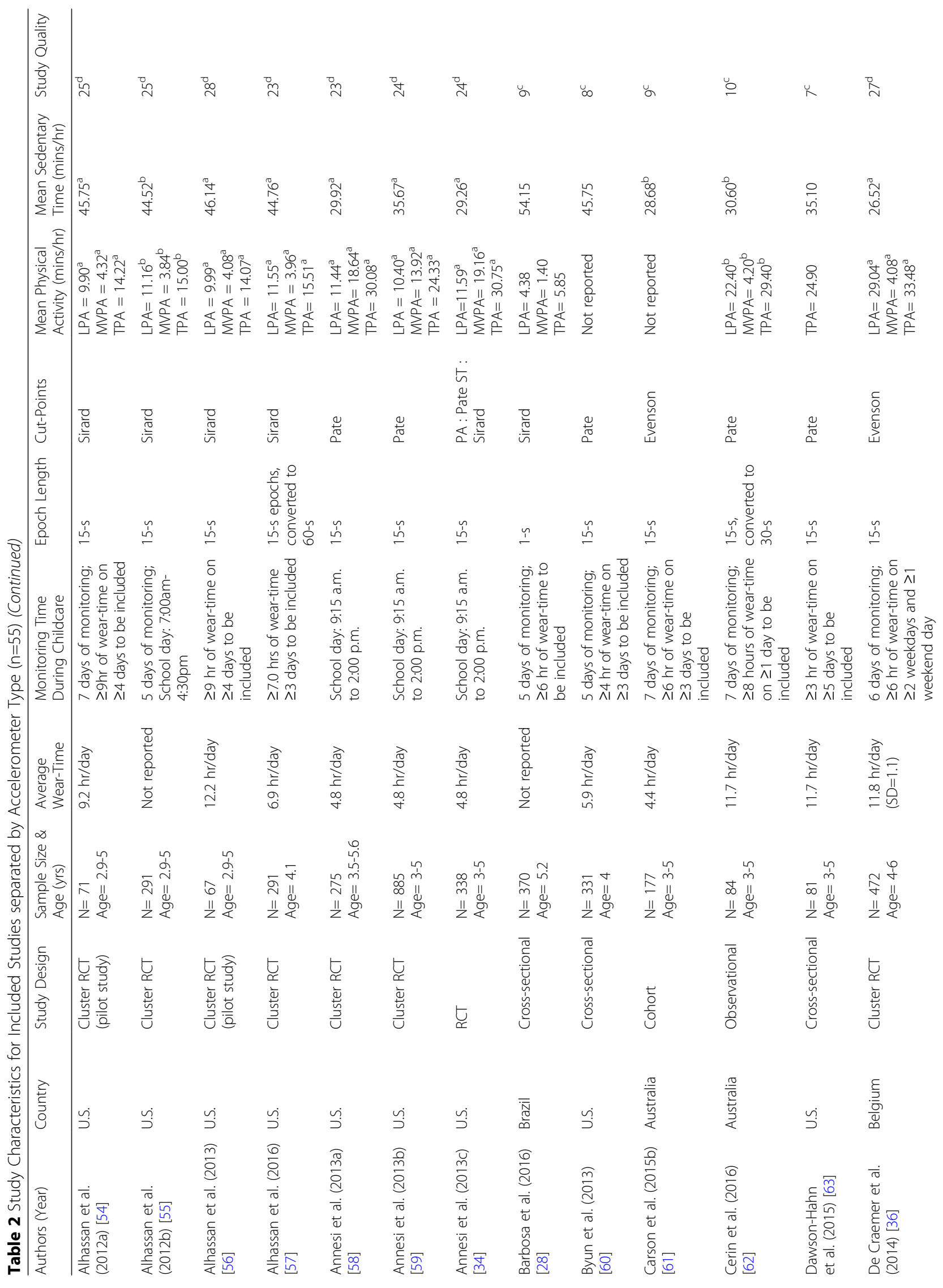




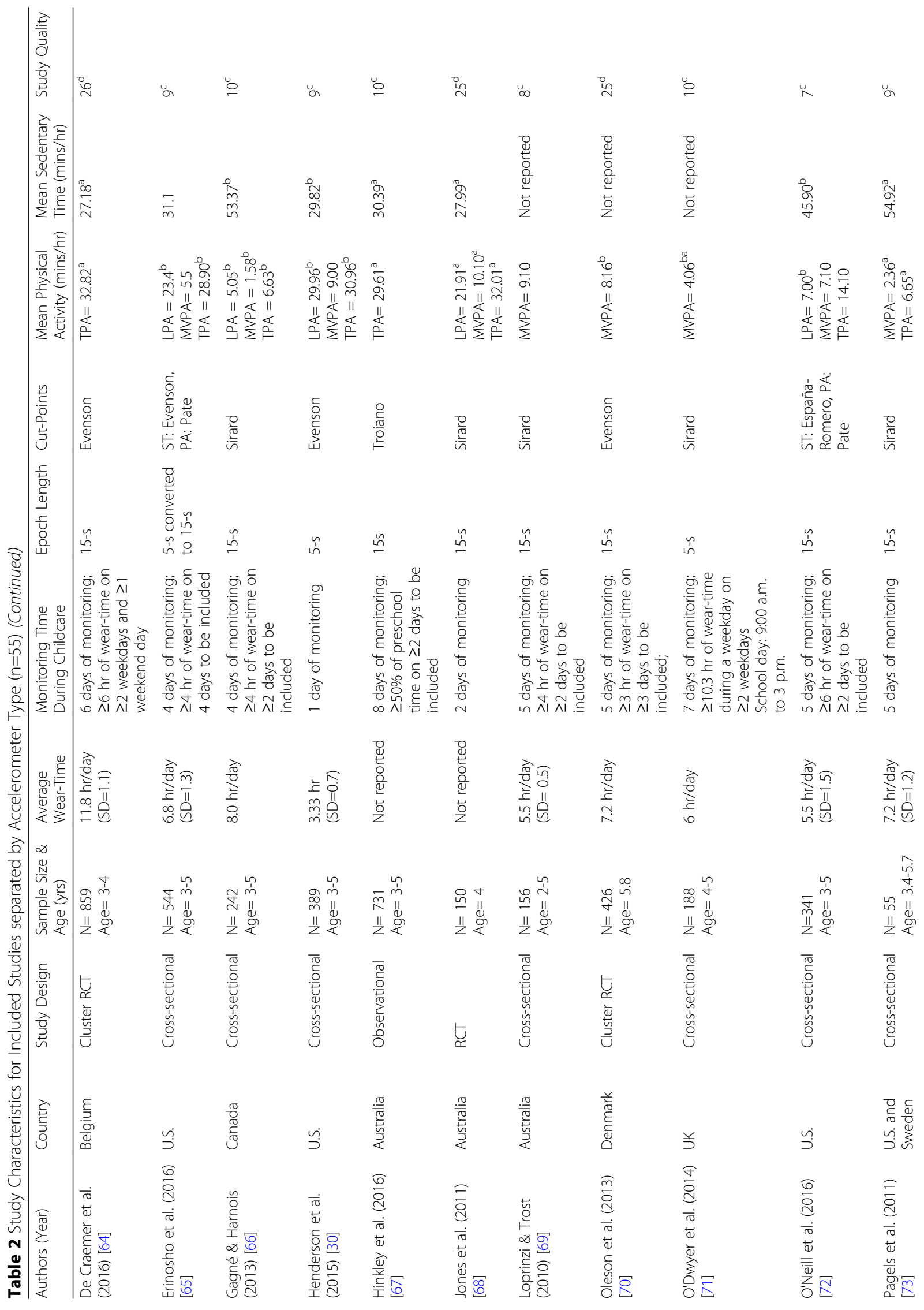




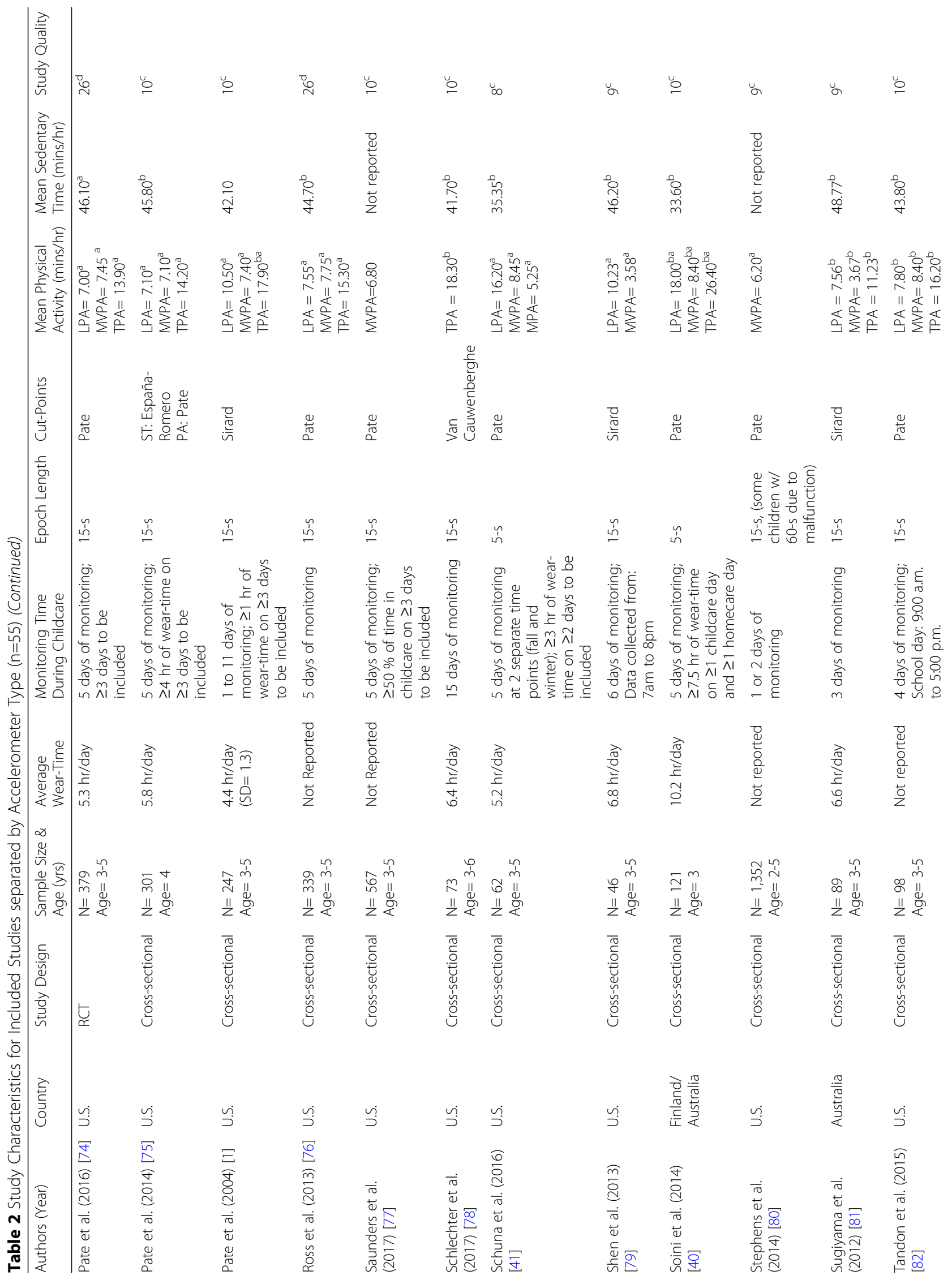




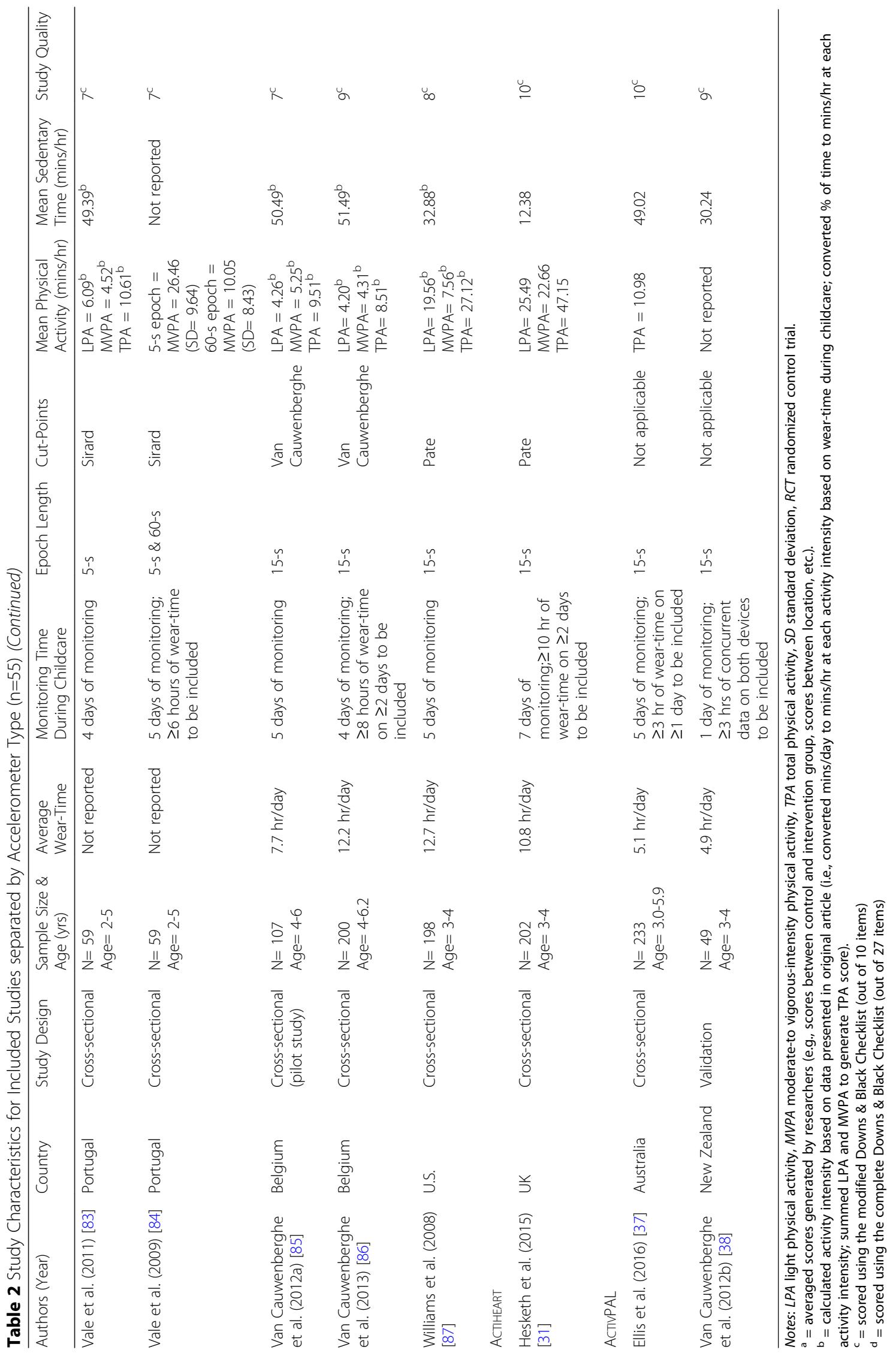


eight articles were retrieved after reviewing the reference lists of included studies, and three via the 'ahead of print' sections from four online journals, bringing the total number of articles to 10,553. Following the removal of duplicates and pre-screening for articles that did not focus on healthy young children, 1,274 articles underwent title and abstract screening by two independent reviewers. Subsequently, full-text review was completed for the remaining 245 articles, with 190 being excluded, leaving 55 articles to be included in the review. See Fig. 1 for the PRISMA flow diagram.

\section{Characteristics of Included Studies}

Included studies were conducted in 11 countries (United States [ $n=28]$, Canada [ $n=8]$, Australia [ $n=8]$, United Kingdom [ $n=2]$, Belgium [ $n=4]$, Denmark $[n=1]$, Portugal $[n=2]$, New Zealand $[n=2]$, Sweden $[n=1]$, Brazil $[n=1]$ and Finland $[n=1])$, with one study conducted in both the United States and Sweden, and another study in both Finland and Australia. The sample size of included articles ranged from 31 to 1,352 , with a mean sample size of 254 young children; the 55 included studies represented a total of 13,956 participants. Date of publication ranged from 2004 to 2017 , with the majority of articles $(n=32,58.2 \%)$ published between 2014-2017. A variety of study designs were employed, the most common being cross-sectional $[n=35]$ and randomized controlled trial $[n=14]$. Both physical activity and sedentary time were measured in 43 of the studies, while the remaining 12 studies measured physical activity $(n=8)$ or sedentary time $(n=4)$ only. A summary of the characteristics of included studies can be found in Table 3.

A variety of accelerometer models were used (Actical $[n=9]$, Actiheart $[n=1]$, and ActivPAL $[n=2])$; however, ActiGraph was by far the most common $[n=44]$. Average accelerometer wear-time ranged from 4.3 [26] to 13.0 [27] hrs/day with monitoring ranging from 1 to 11 days. Most studies used a 15-s epoch, 1[28], with observations as low as 1 -s and 5-s. A variety of cut-points were applied in the various studies with the most frequently adopted including Pate et al. [ $n=19]$, Sirard et al. [ $n=17]$, Pfeiffer et al. [ $n=6$ ], Evenson et al. [ $n=7]$, and Van Cauwenberghe et al. [ $n=3$ ]. See Table 2 for complete study characteristics and outcome data.

\section{Physical Activity Prevalence Rates (LPA, MVPA, TPA)}

Young children's LPA ranged from: 15.88 [19] to 21.53 [29] mins/hr ( $M=18.56)$ for Actical accelerometers; and

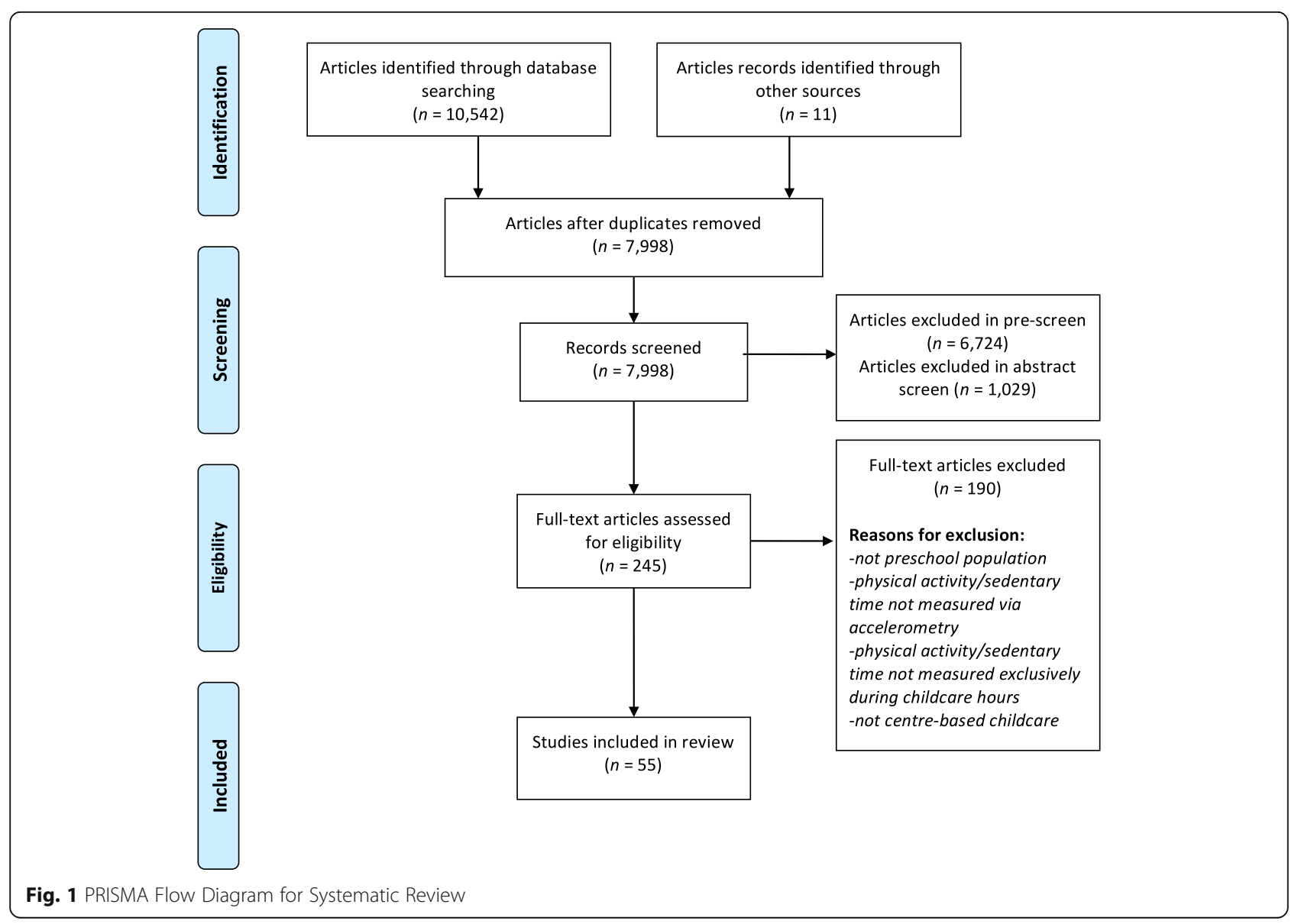


Table 3 Summary of Characteristics for Included Studies

\begin{tabular}{|c|c|}
\hline & \# of studies \\
\hline Years of publication (range) & $2004-2017$ \\
\hline Sample size (\# of participants per study) & $31-1,352$ \\
\hline \multicolumn{2}{|l|}{ Country } \\
\hline United States & 28 \\
\hline Canada & 8 \\
\hline Australia & 8 \\
\hline United Kingdom & 2 \\
\hline Belgium & 4 \\
\hline Denmark & 1 \\
\hline Portugal & 2 \\
\hline New Zealand & 2 \\
\hline Sweden & 1 \\
\hline Brazil & 1 \\
\hline Finland & 1 \\
\hline \multicolumn{2}{|l|}{ Study Design } \\
\hline Cross-sectional & 35 \\
\hline Randomized controlled trial & 14 \\
\hline Cohort & 2 \\
\hline Longitudinal & 1 \\
\hline Validation & 2 \\
\hline Observational & 2 \\
\hline \multicolumn{2}{|l|}{ Accelerometer model } \\
\hline Actical & 9 \\
\hline Actiheart & 1 \\
\hline ActivPAL & 2 \\
\hline ActiGraph & 44 \\
\hline \multicolumn{2}{|l|}{ Cut-points used } \\
\hline Pate et al. & 19 \\
\hline Sirard et al. & 17 \\
\hline Pfeiffer et al. & 6 \\
\hline Evenson et al. & 7 \\
\hline Van Cauwenberghe et al. & 3 \\
\hline
\end{tabular}

2.94 [27] to 29.96 [30] mins/hr $(M=11.80)$ for ActiGraph accelerometers. The one study which used Actiheart accelerometers reported $25.49 \mathrm{mins} / \mathrm{hr}$ of LPA. [31] LPA was not reported for the studies which used ActivPAL devices.

Rates of MVPA were lower for all accelerometer types, ranging from: 1.37 [32] to 5.30 [33] $\mathrm{mins} / \mathrm{hr}(M=2.91)$ for Actical accelerometers; and 1.29 [27] to 19.16 [34] mins/hr $(M=6.67)$ for ActiGraph accelerometers. The one study which used Actiheart accelerometers reported 22.66 [31] mins/hr of MVPA. MVPA was not reported for the two studies which used ActivPAL devices. While observed rates of MVPA were lower than LPA, the rates of MVPA measured via Actical were noticeably lower than those captured via ActiGraph (which produced a $17.87 \mathrm{mins} / \mathrm{hr}$ dispersion), with Actiheart accelerometer producing the highest MVPA score.

TPA accumulated by preschoolers in centre-based childcare ranged from 17.42 [19] to 26.00 [35] $\mathrm{mins} / \mathrm{hr}(M=$ 21.64) for Actical accelerometers; and 4.23 [27] to 33.48 [36] $\mathrm{mins} / \mathrm{hr}(M=18.42)$ for ActiGraph accelerometers. TPA was 47.17 [31] mins/hr for the one study which used Actiheart accelerometers, and 10.98 [37] $\mathrm{mins} / \mathrm{hr}$ for the study which used ActivPAL accelerometry. Figures 2 (ActiGraph) and 3 (Actical and Actiheart) illustrate the hourly rates of physical activity by applied cut-point. These figures illustrate that, generally speaking, studies that employed the same cut-points had hourly rates of MVPA that were comparable.

As 11 countries are represented across this review, activity levels were also analyzed across four geographic regions: North America, South America, Europe and South Pacific (i.e. Australia and New Zealand). Of note, only one study originated from South America (Brazil). Mean LPA was very similar between North America and Europe (13.06 and $14.51 \mathrm{mins} / \mathrm{hr}$, respectively); however, the preschoolers in the South Pacific region seemed to have accumulated more LPA $(M=17.46 \mathrm{mins} / \mathrm{hr})$, while those in the South American study achieved much less (4.38[28] mins/hr). In terms of mean MVPA, the North American and South Pacific regions were quite comparable (6.13 and $7.09 \mathrm{mins} / \mathrm{hr}$, respectively). Mean MVPA across the European studies was slightly higher at 9.12 mins/hr, while the South American study was quite low in comparison (1.40 [28] mins/hr). When considering the mean TPA across studies, preschoolers in the South Pacific region appear to be the most active $(M=23.27 \mathrm{mins} / \mathrm{hr})$, followed by Europe $(M=21.89$ mins/hr), North America $(M=19.57 \mathrm{mins} / \mathrm{hr})$, and South America (5.85 [28] mins/hr).

\section{Sedentary Time Prevalence Rates}

Figures 4 (ActiGraph) and 5 (Actical and Actiheart) display the hourly rates of sedentary time by cut-point applied which illustrates that studies using the same cut-points cluster together. The hourly rates of sedentary time ranged from: 30.61 [38] to 42.38 [32] $\mathrm{mins} / \mathrm{hr}(M=$ 36.47) for Actical accelerometers; 26.52 [36] to 55.77 [27] $\mathrm{mins} / \mathrm{hr}(M=40.88)$ for ActiGraph accelerometers; and 30.24 [38] to 49.02 [37] mins/hr $(M=39.63)$ for ActivPAL accelerometers. The one study that used Actiheart accelerometers reported 12.38 [31] mins/hr of sedentary time.

In exploring sedentary time by geographic region, North American preschoolers appeared to be much more sedentary than those from the Pacific region (41.23 versus 34.43 mins/hr). European studies reported a mean sedentary 


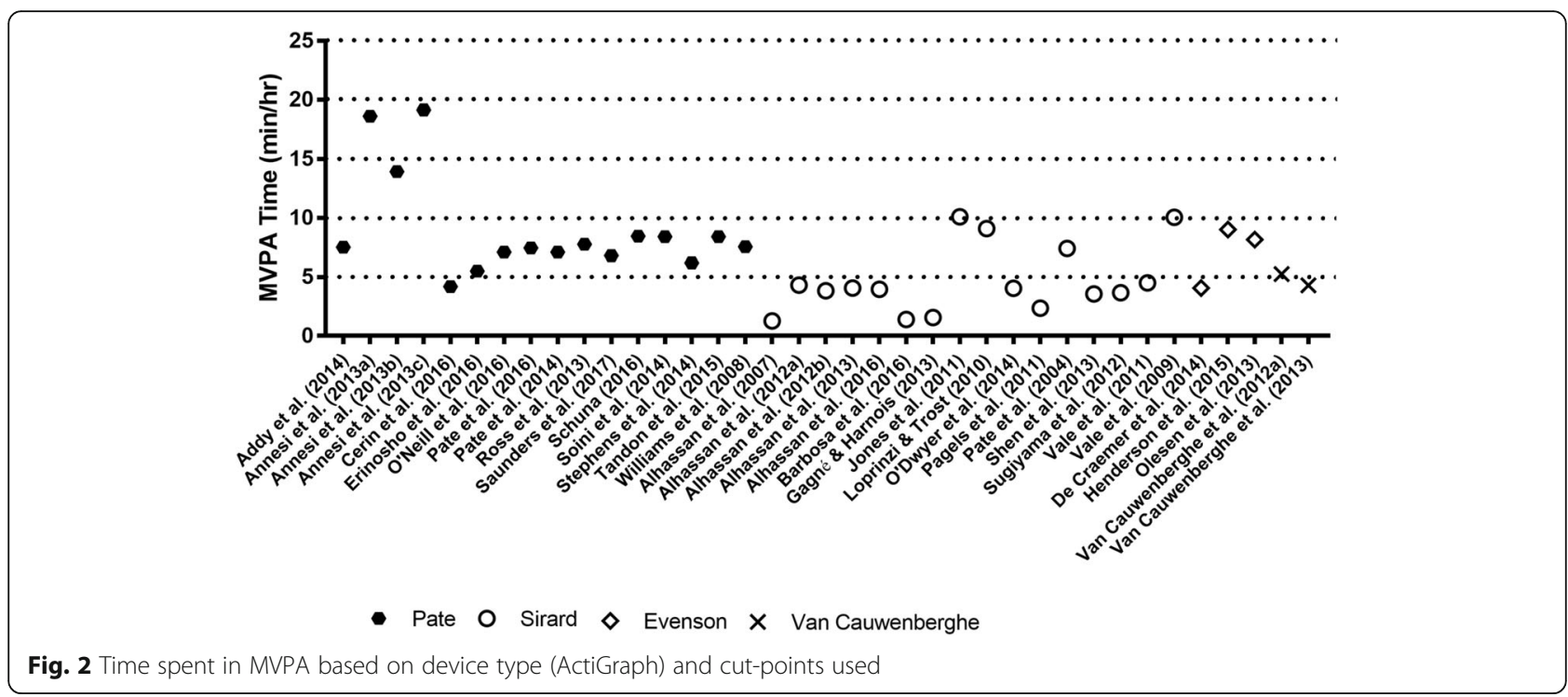

time that was intermediate to these two regions at 38.25 mins/hr. The sole South American study reported preschoolers' sedentary time to be 54.15 [28] mins/hr.

\section{Discussion}

The purpose of this systematic review was to gain a clearer picture of young children's physical activity and sedentary time in centre-based childcare. This paper offers the first synthesis of objectively-measured physical activity and sedentary time in this unique setting among preschoolers. Given the dramatic increase in studies

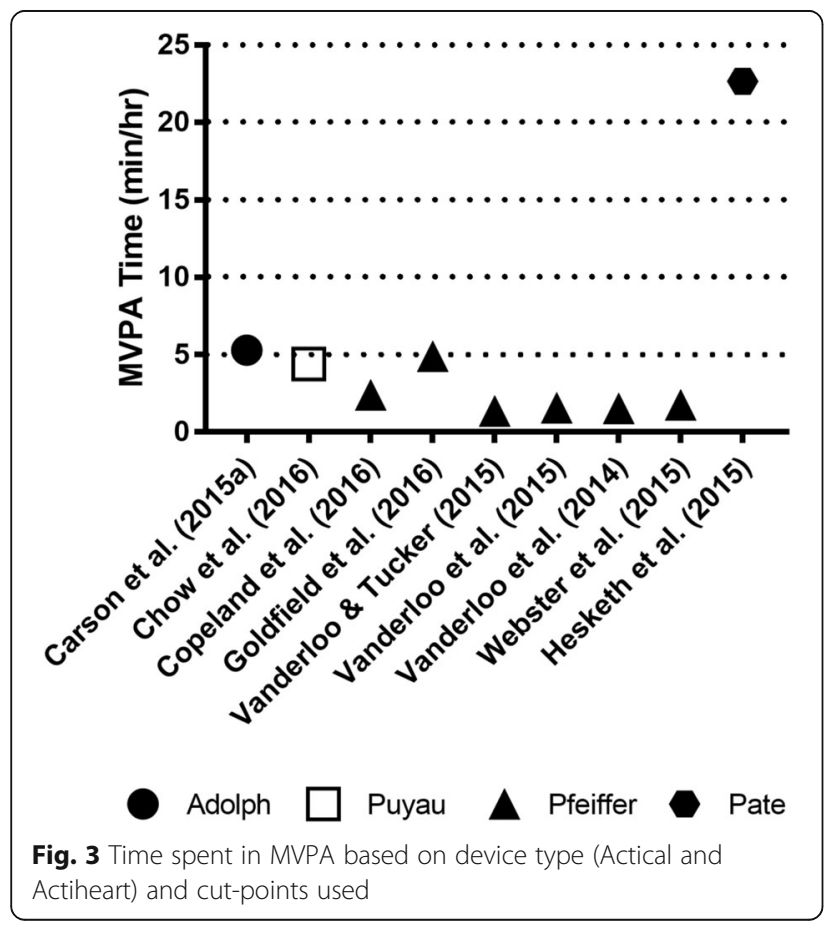

conducted in this environment and with this population, coupled with the ongoing challenges of comparing studies using different accelerometers, this study provides a snapshot of current rates of physical activity and sedentary time captured in centre-based childcare.

The results are highly variable, reporting that physical activity in childcare ranged from 2.94 [27] to 29.96 [30] mins/hr for LPA; 1.29 [27] to 22.66 [31] $\mathrm{mins} / \mathrm{hr}$ for MVPA; and, 4.23 [27] to 47.17 [31] mins/hr for TPA, respectively. There are a number of reasons why these wide ranges were observed, namely, the different accelerometer devices and the cut-points used. The challenge of gathering an accurate picture of activity levels in centre-based childcare is also compounded by the diversity in data collection protocols (i.e., wear time criteria; trying to gather a habitual daily activity level), the output data produced and interpreted; (i.e., mins/hr, counts/ min, \%/wear time), and the variability in childcare centres (which has been shown to be a strong predictor of physical activity levels in this population [39]). With this in mind, Finn and colleagues noted that $50 \%$ of the variation in physical activity levels is a consequence of the childcare environment itself [39]; therefore, the wide ranges observed could also be a reflection of the varying quality of centre-based childcare facilities. As such, all these factors could be contributing to the varied magnitude in physical activity levels, and similarly sedentary time, observed.

Of note, 11 countries were represented in this review. Given different cultural norms and childcare practices, it is possible that geographic region may also influence inter-childcare centre variation. For example, in comparing the mean TPA reported by studies from the South Pacific region (Australia and New Zealand) versus North America, there is over $3.5 \mathrm{mins} / \mathrm{hr}$ difference in TPA 


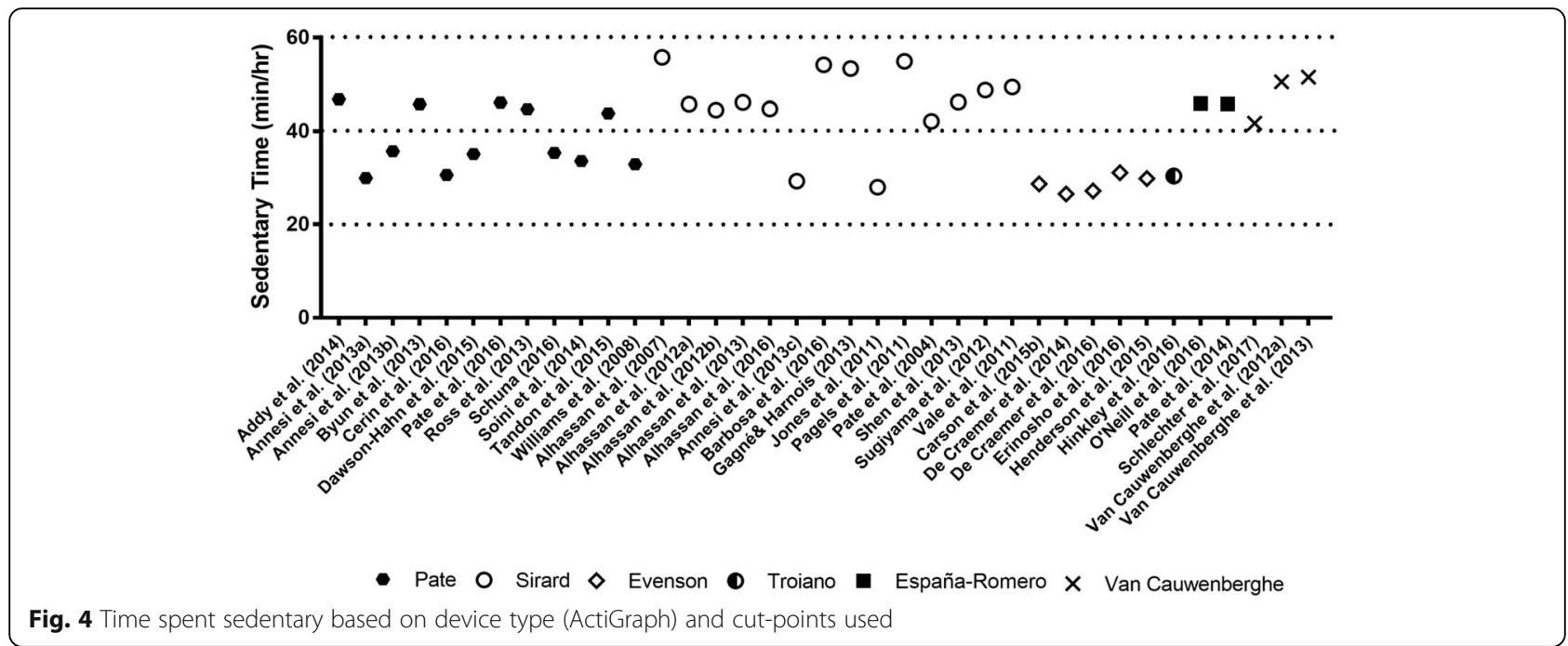

(23.27 versus $19.57 \mathrm{mins} / \mathrm{hr}$ ). When extrapolating these hourly rates over a full childcare day, this difference in activity is substantial. Furthermore, Soini et al. assessed the physical activity levels of 3-year-olds in childcare in both Finland and Australia [40]. Although they did not find inter-country differences in preschoolers' activity levels, they describe various facets of the childcare centres from each country, for example structure of a typical day in childcare and its governance. Soini and colleagues also noted the ranges in outdoor temperature that were experienced

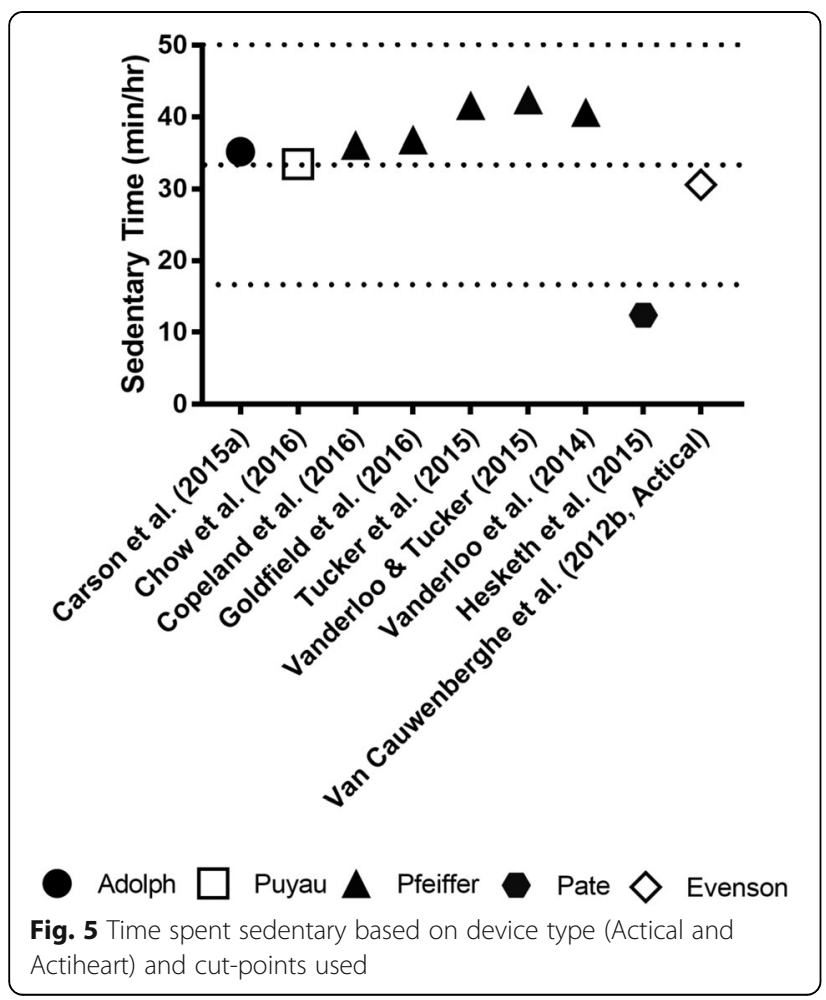

throughout their study [40]. In a review as large as this, it is important to bear in mind that differences in variable such as governance, childcare structure and environment across countries could have an impact on the variation in reported activity levels. Seasonal differences between the countries included in this review could also play a role in producing a wide range of reported activity levels, as temperature differences could affect access to outdoor playtime, which is strongly correlated with activity levels among young children [12, 41, 42].

To clarify the ongoing challenge of comparing physical activity data from different accelerometer models, a recent study explored the differences in physical activity and sedentary time among preschoolers when administering Actical and ActiGraph accelerometer protocols. Specifically, Vanderloo and colleagues found that these two widely used devices, although validated by the same research team and protocols, capture different activity levels at 15- and 60-s epoch lengths [43]. This group determined that the Actical accelerometer reported higher levels of sedentary time, while the ActiGraph device captured more physical activity [43]. Likewise, although Borghese and colleagues noted good agreement between Actical and ActiGraph accelerometers in assessing older children's MVPA levels (9-11 years), they also recognized the need to exercise caution when comparing across devices as reported activity levels are highly contingent on data reduction protocol and cut-points used [44]. These findings are supported in the current study as a greater variety of cut-points were applied in ActiGraphadopted studies, and the rates observed were much wider. In an effort to produce more comparably measured and analyzed data, and therefore, providing a more consistent representation of young children's physical activity levels, a uniform protocol for processing accelerometry data is needed. 
This review confirms that interpreting preschoolers' actual physical activity levels is challenging. Though not specific to the childcare environment, similar issues with variability of results were noted by Hnatiuk et al. in their review of objectively-measured activity levels in preschoolers (as measured by accelerometers, heart rate monitoring, and direct observation) [3]. While the goal of their review was not to explore activity levels in a particular setting, like childcare, they too highlighted the measurement challenges discussed above as impeding their ability to generate a "true" consensus on physical activity levels and sedentary time of preschool-age children [3]. This lack of interpretability makes it very difficult to determine whether young children are accumulating adequate time in physical activity during childcare hours to aid them in meeting the 24-Hour Movement Guidelines adopted by many countries [5-7]. With TPA values in this review ranging from 4.23 [27] to 47.17 [31] mins/hr, and with the assumption that two-thirds of their waking hours are spent in this childcare environment, $[45,46]$ it is unclear whether preschoolers would attain recommended minutes of physical activity [7]. For example, using the lower limit, young children would acquire 33.84 minutes in TPA during childcare hours, while the upper end would engage in 377.12 minutes during that same timeframe. Given the 24-hour Movement Guidelines for the Early Years encourage 180 minutes of TPA per day, [7] many children would be far surpassing this expectation, while some are well below it. Estimating MVPA time in line with the guidelines (i.e., 60 minutes per day) is equally ominous as rates ranged from 11.12 to 181.29 minutes during the childcare day [8]. Bornstein and colleagues in their 2011 meta-analysis reported a rate of 42.8 minutes per day of MVPA [47]. While not specific to the childcare setting, they too highlight the need for careful consideration when interpreting physical activity levels among this population and that steps are warranted to unify accelerometer-generated physical activity data to inform unbiased and improved comparisons across studies. Though some may perceive this inability to synthesize a univariate finding from the published data as a limitation, the present authors view this as a reflection of the lack of homogeneity in measuring, processing, and reporting objective physical activity data in the literature, while also bearing in mind the impact of factors such variation in childcare centre characteristics [39].

Similar to physical activity, reported sedentary time was mixed, though much higher levels of this behaviour were registered across studies. Specifically, time spent being sedentary ranged from 12.38 [31] to 55.77 [27] mins/hr. These findings could likely be attributed to the fact that the childcare environment is oftentimes referred to as a sedentary and/or obesogenic setting. Past work [2, 19, 48] highlight opportunities for sedentary behaviours (e.g. access to screens in childcare, etc.) as key contributors to high levels of this deleterious health behaviour. Limited outdoor space for free play and gross motor movement, combined with safety and liability concerns, may also be contributing factors worth considering. The types of sedentary behaviour may differ between geographic regions; however, this review did not measure behaviour types, as the focus was time spent in this intensity. This finding is in line with a previous review conducted by our research team in home- or family-based childcare, [20] where the results also documented low levels of physical activity and high levels of sedentary time among preschoolers. While these wide ranges are again, inhibiting a true depiction of sedentary levels among preschoolers in childcare, what is apparent is the higher rates noted, especially compared to MVPA. This has been confirmed elsewhere - Ellis et al. (2017) reported that preschoolers in childcare spend $48.4 \%$ of their time sitting, while only $19.1 \%$ in physical activity [37].

\section{Strengths and Limitations}

The study provides a comprehensive synthesis of all studies measuring physical activity and sedentary time in centre-based childcare settings. In addition to the sheer magnitude of literature included, the review has provided rates of different activity levels within the context of the primary objective measurement techniques within this population. Despite efforts to provide meaningful data regarding activity levels in childcare, via mean hourly rates separated by accelerometer brand and examined across intensities, the methodological variations adopted between studies produced wide discrepancies in activity data. As such, a primary limitation of this review is the inability to provide a consistent picture of young children's physical activity levels in childcare due to discrepancies or inconsistencies in how such data is collected and reported across published work. However, as previously discussed, this lack of clarity regarding young children's activity levels also serves as an important finding where additional work is needed to address this knowledge gap. Second, while all studies were considered "high quality" based on the Downs and Black tool, [23] this assessment could not account for accelerometry protocol approaches and subsequent reporting [49]. The adoption of consistent study protocols (i.e., use of similar accelerometers, cut-points, and data reduction techniques) could potentially allow for this conclusion to be determined, and represents an ongoing challenge in the physical activity literature. Heightened attention should be paid to ideal accelerometer wear time and time spent monitoring children during childcare, as this could affect study quality. It is difficult, for 
example, to compare results of a study that monitored participants for one day in childcare with 7 hours of accelerometer wear time against a study where preschoolers wore accelerometers for 5 hours each day, yet were monitored for a week. Third, only English articles were captured in this review, thus relevant studies published in other languages may have been missed. Lastly, numerous articles in this review did not report participants' TPA within their results, and though it could be calculated when adequate information was provided, reporting TPA values are important given the target outlined by current international movement guidelines $[6,7,50]$.

\section{Conclusion}

It was difficult to ascertain a consistent representation of this population's activity levels due to inconsistencies in measurement approaches used in the literature. Factors related to childcare centre characteristics, as well as the geographical locations where the studies were conducted may also have contributed to this variation. While recognizing the disparities across included studies, it did appear that sedentary time, in comparison to MVPA, was high. Consistent and appropriate accelerometry protocols are essential to gain insight into the levels of activity and inactivity in centre-based childcare, and to help gain an accurate picture of the proportion of children meeting (or not) the new international 24-Hour Movement Guidelines. Such information could also further enable the creation and support of appropriate policies for this environment and may help to create a healthier daily experience for preschoolers.

\section{Abbreviations}

LPA: Light physical activity; MVPA: Moderate-to-vigorous physical activity; PRISMA: Preferred Reporting Items for Systematic Reviews and Meta-

Analyses; RCT: Randomized controlled trial; TPA: Total physical activity

\section{Acknowledgements}

The authors would like to thank Marisa Tippett (Research and Instructional Services Librarian, University of Western Ontario) for her assistance in developing the current review's search strategy. Vanderloo was supported by the CIHR Frederick Banting and Charles Best Doctoral Research Award (2014-2016) and Fellowship Award (2017-2018); Bruijns and Truelove were supported by the Ontario Graduate Scholarship (2016-2017); and Tucker was supported by an Early Researcher Award from the Ontario Ministry of Research and Innovation.

\section{Funding}

There is no funding to declare of this study.

\section{Availability of data and materials}

The dataset generated and analysed during the current study is available from the corresponding author on reasonable request.

\section{Authors' contributions}

PT and LMV conceived and designed the study and were involved with all aspects of this research. KTO, BAB, and ST led data collection and analysis. All authors assisted with the interpretation of the results. KTO and LMV drafted the manuscript, and all authors provided important intellectual contributions to the final document.
Ethics approval and consent to participate

This study did not require ethical approval.

Consent for publication

Not applicable.

Competing interests

The authors declare that they have no competing interests.

\section{Publisher's Note}

Springer Nature remains neutral with regard to jurisdictional claims in published maps and institutional affiliations.

\section{Author details}

'Department of French Studies, Faculty of Arts and Humanities, University of Western Ontario, London, Ontario, Canada. ${ }^{2}$ Child Health Evaluative Sciences, The Hospital for Sick Children, Toronto, Ontario, Canada. ${ }^{3}$ Health and Rehabilitation Sciences, Faculty of Health Sciences, University of Western Ontario, London, Ontario, Canada. ${ }^{4}$ School of Occupational Therapy, Faculty of Health Sciences, University of Western Ontario, 1201 Western Road, Elborn College, Room 2547, London N6G 1H1, Ontario, Canada.

Received: 11 May 2018 Accepted: 30 October 2018

Published online: 21 November 2018

References

1. Pate RR, Pfeiffer KA, Trost SG, Ziegler P, Dowda M. Physical Activity Among Children Attending Preschools. Pediatrics. 2004;114:1258-63. https://doi.org/ 10.1542/peds.2003-1088-L.

2. Tucker P, Vanderloo LM, Burke SM, Irwin JD, Johnson AM. Prevalence and influences of preschoolers' sedentary behaviors in early learning centers: a cross-sectional study. BMC Pediatr. 2015;15:128. https://doi.org/10.1186/ s12887-015-0441-5.

3. Hnatiuk JA, Salmon J, Hinkley T, Okely AD, Trost S. A review of preschool children's physical activity and sedentary time using objective measures. Am J Prev Med. 2014;47(4):487-97. https://doi.org/10.1016/j. ampere.2014.05.042

4. Raustorp A, Pagels P, Boldemann C, Cosco N, Soderstrom M, Martensson F. Accelerometer measured level of physical activity indoors and outdoors during preschool time in Sweden and the United States. J Phys Act Health. 2012;9(6):801-8 doi.org/10.1123/jpah.9.6.801.

5. Ministry of Health. Sit Less, Move More, Sleep Well: Active play guidelines for under-fives. 2017. Retreived from: https://www.health.govt.nz/ publication/sit-less-move-more-sleep-well-active-play-guidelines-under-fives.

6. Okely AD, Ghersi D, Hesketh KD, Santos R, Loughran SP, Cliff DP, et al. A collaborative approach to adopting/adapting guidelines-The Australian 24Hour Movement Guidelines for the early years (Birth to 5 years): an integration of physical activity, sedentary behavior, and sleep. BMC Public Health. 2017;17:869. https://doi.org/10.1186/s12889-017-4867-6.

7. Tremblay MS, Chaput J-P, Adamo KB, Aubert S, Barnes JD, Choquette L, et al. Canadian 24-Hour Movement Guidelines for the Early Years (0-4 years): An Integration of Physical Activity, Sedentary Behaviour, and Sleep. BMC Public Health. 2017;17(5):874; doi.org.10.1186/s12889-017-4859-6.

8. Tremblay MS, Carson V, Chaput J-P, Connor Gorber S, Dinh T, Duggan M, et al. Canadian 24-hour movement guidelines for children and youth: an integration of physical activity, sedentary behaviour, and sleep. Appl Physiol Nutr Metab. 2016;41(6 3):S311-27. https://doi.org/10.1139/apnm-2016-0151.

9. Cleveland G, Forer B, Hyatt D, Japel C, Krashinsky M. New Evidence about Child Care in Canada: Use Patterns, Affordability, and Quality. IRPP Choices. 2008;14:12.

10. Sinha M. Child care in Canada. 2014:1-11. Retreived from: https://www150. statcan.gc.ca/n1/pub/89-652-x/89-652-x2014005-eng.pdf

11. Vanderloo LM, Tucker P, Johnson AM, Burke SM, Irwin JD. Environmenta Influences on Preschoolers' Physical Activity Levels in Various Early-Learning Facilities. Res Q Exerc Sport. 2015;86(4):360-70. https://doi.org/10.1080/ 02701367.2015.1053105.

12. Mazzucca S, Hales D, Evenson KR, Ammerman A, Tate DF, Berry DC, et al. Physical Activity Opportunities Within the Schedule of Early Care and Education Centers. J Phys Act Health. 2018;15(2):73-81. https://doi.org/10. 1123/jpah.2017-0071. 
13. Bower JK, Hales DP, Tate DF, Rubin DA, Benjamin SE, Ward DS. The childcare environment and children's physical activity. Am J Prev Med. 2008; 34(1):23-9. https://doi.org/10.1016/j.amepre.2007.09.022.

14. Oliver M, Schofield G, Kolt G. Physical activity in preschoolers: understanding prevalence and measurement issues. Sport Med. 2007;37(12):1045-70. https://doi.org/10.2165/00007256-200737120-00004.

15. Reilly JJ. Physical activity, sedentary behaviour and energy balance in the preschool child: opportunities for early obesity prevention. Proc Nutr Soc. 2008;67(3):317-25. https://doi.org/10.1017/S0029665108008604.

16. Tucker $P$. The physical activity levels of preschool-aged children: $A$ systematic review. Early Child Res Q. 2008;23(4):547-58. https://doi.org/10. 1016/j.ecresq.2008.08.005

17. Ward DS, Vaughn A, McWilliams C, Hales DP. Interventions for increasing physical activity at child care. Med Sci Sports Exerc. 2010;42(3):526-34. https://doi.org/10.1249/MSS.0b013e3181cea406.

18. Cliff DP, Reilly JJ, Okely AD. Methodological considerations in using accelerometers to assess habitual physical activity in children aged 0-5 years. J Sci Med Sport. 2009;12(5):557-67. https://doi.org/10.1016/j.jsams. 2008.10.008.

19. Vanderloo LM, Tucker $P$, Johnson AM, van Zandvoort MM, Burke SM, Irwin JD. The influence of centre-based childcare on preschoolers' physical activity levels: a cross-sectional study. Int J Environ Res Public Health. 2014 11(2):1794-802. https://doi.org/10.3390/ijerph110201794.

20. Vanderloo LM, Martyniuk OJM, Tucker P. Physical and Sedentary Activity Levels Among Preschoolers in Home-Based Childcare: A Systematic Review. J Phys Act Health. 2015;12(6):879-89. https://doi.org/10.1123/jpah.2013-0483.

21. Liberati A, Altman DG, Tetzlaff J, Mulrow C, Gøtzsche PC, loannidis JPA, et al. The PRISMA statement for reporting systematic reviews and metaanalyses of studies that evaluate health care interventions: explanation and elaboration. PLoS Med. 2009;6(7):e1000100 doi.org/10.1371/journal. pmed. 1000100 .

22. Moher D, Shamseer L, Clarke M, Ghersi D, Liberati A, Petticrew $M$, et al. Preferred reporting items for systematic review and meta-analysis protocols (PRISMA-P) 2015 statement. Syst Rev. 2015;4:1. https://doi.org/ 10.1186/2046-4053-4-1.

23. Downs $\mathrm{SH}$, Black N. The feasibility of creating a checklist for the assessment of the methodological quality both of randomised and non-randomised studies of health care interventions. J Epidemiol Community Health. 1998; 52(6):377-84.

24. Vanderloo LM. Screen-viewing among preschoolers in childcare: A systematic review. BMC Pediatr. 2014;14(205). https://doi.org/10.1186/1471-2431-14-205.

25. Duch $\mathrm{H}$, Fisher EM, Ensari I, Harrington A. Screen time use in children under 3 years old: A systematic review of correlates. Int J Behav Nutr Phys Act. 2013;10(102). https://doi.org/10.1186/1479-5868-10-102

26. Webster EK, Wadsworth DD, Robinson LE. Preschoolers' time on-task and physical activity during a classroom activity break. Pediatr Exerc Sci. 2015; 27(1):160-7. https://doi.org/10.1123/pes.2014-0006.

27. Alhassan S, Sirard JR, Robinson TN. The effects of increasing outdoor play time on physical activity in Latino preschool children. Pediatr Obes. 2007; 2(3):153-8. https://doi.org/10.1080/17477160701520108

28. Barbosa SC, Coledam DHC, Stabelini Neto A, Elias RGM, de Oliveira AR. School environment, sedentary behavior and physical activity in preschool children. Rev Paul Pediatr. 2016;34(3):301-8. https://doi.org/10. 1016/j.rppede.2016.02.003

29. Copeland KA, Khoury JC, Kalkwarf HJ. Child Care Center Characteristics Associated With Preschoolers' Physical Activity. Am J Prev Med. 2016;50(4): 470-9. https://doi.org/10.1016/j.amepre.2015.08.028.

30. Henderson KE, Grode GM, O'Connell ML, Schwartz MB. Environmental factors associated with physical activity in childcare centers. Int J Behav Nutr Phys Act. 2015;12(43). https://doi.org/10.1186/s12966-015-0198-0.

31. Hesketh KR, Griffin SJ, van Sluijs EMF. UK Preschool-aged children's physical activity levels in childcare and at home: a cross-sectional exploration. Int J Behav Nutr Phys Act. 2015;12(123). https://doi.org/10. 1186/s12966-015-0286-1.

32. Vanderloo LM, Tucker P. Weekly trends in preschoolers' physical activity and sedentary time in childcare. Int J Environ Res Public Health. 2015;12(3):245464. https://doi.org/10.3390/ijerph120302454.

33. Carson V, Clark D, Ogden N, Harber V, Kuzik N. Short-Term Influence of Revised Provincial Accreditation Standards on Physical Activity, Sedentary Behavior, and Weight Status in Alberta, Canada Child Care Centers. Early Child Educ J. 2015;43(6):459-65. https://doi.org/10.1007/s10643-015-0688-3.
34. Annesi JJ, Smith AE, Tennant GA. Effects of the Start For Life treatment on physical activity in primarily African American preschool children of ages 3 5 years. Psychol Health Med. 2013;18(3):300-9. https://doi.org/10.1080/ 13548506.2012.712704.

35. Chow AF, Leis A, Humbert L, Muhajarine N, Engler-Stringer R. Healthy Start-Départ Santé: A pilot study of a multilevel intervention to increase physical activity, fundamental movement skills and healthy eating in rural childcare centres. Can J Public Heal. 2016;107(3):312-8. https://doi.org/10.17269/cjph.107.5279.

36. De Craemer M, De Decker E, Verloigne M, De Bourdeaudhuij I, Manios Y, Cardon G. The effect of a kindergarten-based, family-involved intervention on objectively measured physical activity in Belgian preschool boys and girls of high and low SES: the ToyBox-study. Int J Behav Nutr Phys Act. 2014; 11(38). https://doi.org/10.1186/1479-5868-11-38.

37. Ellis YG, Cliff DP, Janssen X, Jones RA, Reilly JJ, Okely AD. Sedentary time, physical activity and compliance with IOM recommendations in young children at childcare. Prev Med reports. 2017;7:221-6. https://doi.org/10. 1016/j.pmedr.2016.12.009.

38. Van Cauwenberghe E, Wooller L, Mackay L, Cardon G, Oliver M. Comparison of Actical and activPAL measures of sedentary behaviour in preschool children. J Sci Med Sport. 2012;15(6):526-31. https://doi.org/ 10.1016/j.jsams.2012.03.014.

39. Finn K, Johannsen N, Specker B. Factors associated with physical activity in preschool children. J Pediatr. 2002;140(1):81-5. https://doi.org/10. 1067/mpd.2002.120693.

40. Soini A, Watt A, Tammelin T, Soini M, Sääkslahti A, Poskiparta M. Comparing the physical activity patterns of 3-year-old Finnish and Australian children during childcare and homecare days. Balt J Heal Phys Act. 2014;6(3):171-82. https://doi.org/10.2478/bjha-2014-0015.

41. Schuna JM, Liguori G, Tucker JTJ. Seasonal Changes in Preschoolers' Sedentary Time and Physical Activity at Childcare. Int J Child Heal Nutr. 2016;5:17-24. https://doi.org/10.6000/1929-4247.2016.05.01.3.

42. Baranowski T, Thompson WO, Durant RH, Baranowski J, Puhl J. Observations on physical activity in physical locations: Ager gender, ethnicity, and month effects. Res Q Exerc Sport. 1993;64(2):127-33. https://doi.org/10.1080/ 02701367.1993.10608789.

43. Vanderloo LM, Di Cristofaro NA, Proudfoot NA, Tucker P, Timmons BW. Comparing the Actical and ActiGraph Approach to Measuring Young Children's Physical Activity Levels and Sedentary Time. Pediatr Exerc Sci. 2016;28(1):133-42. https://doi.org/10.1123/pes.2014-0218.

44. Borghese MM, Tremblay MS, Leblanc AG, Leduc G, Boyer C, Chaput JP. Comparison of ActiGraph GT3X + and Actical accelerometer data in 9 - 11year-old Canadian children. J Sports Sci. 2017;35(6):517-24. https://doi.org/ 10.1080/02640414.2016.1175653.

45. Snyder TD, Hoffman CM. Digest of Education Statistics 2001. 2002. Retrieved from: https://nces.ed.gov/pubs2002/2002130.pdf

46. Bushnik T. Children and Youth Research Paper Series: Child Care in Canada. 2006.

47. Bornstein DB, Beets MW, Byun W, Mciver K. Accelerometer-derived physical activity levels of preschoolers : A meta-analysis. J Sci Med Sport. 2011;14(6): 504-11. https://doi.org/10.1016/j.jsams.2011.05.007.

48. Gubbels JS, Kremers SPJ, van Kann DHH, Stafleu A, Candel MJJM, Dagnelie $P C$, et al. Interaction between physical environment, social environment, and child characteristics in determining physical activity at child care. Health Psychol. 2011;30(1):84-90. https://doi.org/10.1037/a0021586.

49. Montoye AHK, Moore RW, Bowles HR, Korycinski R, Pfeiffer KA. Reporting accelerometer methods in physical activity intervention studies: a systematic review and recommendations for authors. Br J Sport Med 2016;0: 1-10; doi:https://doi.org/10.1136/bjsports-2015-095947.

50. Department of Health Physical Activity Health Improvement and Protection. Start Active, Stay Active. A report on physical activity for health from the four home countries' Chief Medical Officers. 2011. Retrieved from: https:// assets.publishing.service.gov.uk/government/uploads/system/uploads/ attachment_data/file/216370/dh_128210.pdf

51. Truelove $S$, Bruijns BA, Vanderloo LM, O'Brien KT, Johnson AM, Tucker $P$. Physical activity and sedentary time during childcare outdoor play sessions: A systematic review and meta-analysis. Prev Med. 2018;108:74-85. https:// doi.org/10.1016/j.ypmed.2017.12.022.

52. Goldfield GS, Harvey ALJ, Grattan KP, Temple V, Naylor P-J, Alberga AS, et al. Effects of child care intervention on physical activity and body composition. Am J Prev Med. 2016;51(2):225-31. https://doi.org/10.1016/ j.amepre.2016.03.024. 
53. Addy CL, Trilk JL, Dowda M, Byun W, Pate RR. Assessing preschool children's physical activity: how many days of accelerometry measurement. Pediatr Exerc Sci. 2014;26(1):103-9. https://doi.org/10.1123/pes.2013-0021.

54. Alhassan S, Nwaokelemeh O, Ghazarian M, Roberts J, Mendoza A, Shitole S. Effects of Locomotor Skill Program on Minority Preschoolers' Physical Activity Levels. Pediatr Exerc Sci. 2012;24(3):435-49. https://doi.org/10.1123/ pes.24.3.435

55. Alhassan S, Nwaokelemeh O, Mendoza A, Shitole S, Whitt-Glover MC, Yancey AK. Design and baseline characteristics of the Short bouTs of Exercise for Preschoolers (STEP) study. BMC Public Health. 2012;12(582). https://doi.org/10.1186/1471-2458-12-582.

56. Alhassan S, Nwaokelemeh O, Lyden K, Goldsby T, Mendoza A. A Pilot Study to Examine the Effect of Additional Structured Outdoor Playtime on Preschoolers' Physical Activity Levels. Child Care Pract. 2013;19(1):23-35. https://doi.org/10.1080/13575279.2012.712034

57. Alhassan S, Nwaokelemeh O, Mendoza A, Shitole S, Puleo E, Pfeiffer KA, et al. Feasibility and Effects of Short Activity Breaks for Increasing PreschoolAge Children's Physical Activity Levels. J Sch Health. 2016;86(7):526-33. https://doi.org/10.1111/josh.12403.

58. Annesi JJ, Smith AE, Tennant G. Cognitive-behavioural physical activity treatment in African-American pre-schoolers: effects of age, sex, and BMI. J Paediatr Child Health. 2013;49(2):E128-32. https://doi.org/10.1111/jpc.12082.

59. Annesi JJ, Smith AE, Tennant GA. Effects of a cognitive-behaviorally based physical activity treatment for 4- and 5-year-old children attending US preschools, Int J Behav Med. 2013;20(4):562-6. https://doi.org/10.1007/s12529-013-9361-7.

60. Byun W, Blair SN, Pate RR. Objectively measured sedentary behavior in preschool children: comparison between Montessori and traditional preschools. Int J Behav Nutr Phys Act. 2013;10(2). https://doi.org/10.1186/ 1479-5868-10-2.

61. Carson V, Salmon J, Crawford D, Hinkley T, Hesketh KD. Longitudinal levels and bouts of objectively measured sedentary time among young Australian children in the HAPPY study. J Sci Med Sport. 2016;19(3):232-6. https://doi. org/10.1016/j.jsams.2015.01.009.

62. Cerin E, Baranowski T, Barnett A, Butte N, Hughes $\mathrm{S}$, Lee RE, et al. Places where preschoolers are (in) active: an observational study on Latino preschoolers and their parents using objective measures. Int J Behav Nutr Phys Act. 2016;13(29). https://doi.org/10.1186/s12966-016-0355-0.

63. Dawson-Hahn EE, Fesinmeyer MD, Mendoza JA. Correlates of physical activity in Latino preschool children attending Head Start. Pediatr Exerc Sci. 2015;27(3):372-9. https://doi.org/10.1123/pes.2014-0144.

64. De Craemer M, De Decker E, Verloigne M, De Bourdeaudhuij I, Manios $Y$, Cardon $G$, et al. The effect of a cluster randomised control trial on objectively measured sedentary time and parental reports of time spent in sedentary activities in Belgian preschoolers: the ToyBoxstudy. Int J Behav Nutr Phys Act. 2016;13(1). https://doi.org/10.1186/ s12966-015-0325-y.

65. Erinosho T, Hales D, Vaughn A, Mazzucca S, Ward DS. Impact of Policies on Physical Activity and Screen Time Practices in 50 Child-Care Centers in North Carolina. J Phys Act Health. 2016;13(1):59-66. https://doi.org/10.1123/ jpah.2014-0467.

66. Gagné C, Harnois I. The contribution of psychosocial variables in explaining preschoolers' physical activity. Heal Psychol. 2013;32(6):657-65. https://doi. org/10.1037/a0031638.

67. Hinkley T, Salmon J, Crawford D, Okely AD, Hesketh KD. Preschool and childcare center characteristics associated with children's physical activity during care hours: an observational study. Int J Behav Nutr Phys Act. 2016; 13(117). https://doi.org/10.1186/s12966-016-0444-0.

68. Jones RA, Riethmuller A, Hesketh K, Trezise J, Batterham M, Okely AD. Promoting fundamental movement skill development and physical activity in early childhood settings: a cluster randomized controlled trial. Pediatr Exerc Sci. 2011;23(4):600-15. https://doi.org/10.1123/pes.23.4.600.

69. Loprinzi PD, Trost SG. Parental influences on physical activity behavior in preschool children. Prev Med. 2010;50(3):129-33. https://doi.org/10.1016/j. ypmed.2009.11.010.

70. Olesen LG, Kristensen PL, Korsholm L, Froberg K. Physical activity in children attending preschools. Pediatrics. 2013;132(5):e1310-8. https://doi.org/10. 1542/peds.2012-3961.

71. O'Dwyer M, Fairclough SJ, Ridgers ND, Knowles ZR, Foweather L, Stratton G. Patterns of objectively measured moderate-to-vigorous physical activity in preschool children. J Phys Act Heal. 2014;11(6):1233-8. https://doi.org/10. 1123/jpah.2012-0163.
72. O'Neill JR, Pfeiffer KA, Dowda M, Pate RR. In-school and Out-of-school Physical Activity in Preschool Children. J Phys Act Health. 2016;13(6):606-10. https://doi.org/10.1123/jpah.2015-0245.

73. Pagels P, Boldemann C, Raustorp A. Comparison of pedometer and accelerometer measures of physical activity during preschool time on 3-to 5-year-old children. Acta Paediatr. 2011;100(1):116-20. https://doi.org/10. $1111 / \mathrm{j} .1651-2227.2010 .01962 . x$.

74. Pate RR, Brown WH, Pfeiffer KA, Howie EK, Saunders RP, Addy CL, et al. An intervention to increase physical activity in children: a randomized controlled trial with 4-year-olds in preschools. Am J Prev Med. 2016;51(1): 12-22. https://doi.org/10.1016/j.amepre.2015.12.003.

75. Pate RR, O'Neill JR, Byun W, Mclver KL, Dowda M, Brown WH. Physical activity in preschool children: comparison between Montessori and traditional preschools. J Sch Health. 2014;84(11):716-21. https://doi.org/10. 1111/josh.12207.

76. Taverno Ross S, Dowda M, Saunders R, Pate R. Double Dose: The Cumulative Effect of TV Viewing at Home and in Preschool on Children's Activity Patterns and Weight Status. Pediatr Exerc Sci. 2013;25(2):262-72. https://doi.org/10.1123/pes.25.2.262.

77. Saunders RP, Pfeiffer K, Brown WH, Howie EK, Dowda M, O'Neill JR, et al. Evaluating and Refining the Conceptual Model Used in the Study of Health and Activity in Preschool Environments (SHAPES) Intervention. Heal Educ Behav. 2017;44(6):876-84. https://doi.org/10.1177/1090198116686334.

78. Schlechter CR, Rosenkranz RR, Fees BS, Dzewaltowski DA. Preschool daily patterns of physical activity driven by location and social context. J Sch Health. 2017;87(3):194-9. https://doi.org/10.1111/josh.12486.

79. Shen B, Alexander G, Milberger S, Jen K-LC. An exploratory study of seasonality and preschoolers' physical activity engagement. J Phys Act Health. 2013;10(7):993-9. https://doi.org/10.1123/jpah.10.7.993.

80. Stephens RL, Xu Y, Lesesne CA, Dunn L, Kakietek J, Jernigan J, et al. Relationship Between Child Care Centers' Compliance With Physical Activity Regulations and Children's Physical Activity, New York City, 2010. Prev Chronic Dis. 2014;11(E179). https://doi.org/10.5888/pcd11.130432.

81. Sugiyama T, Okely AD, Masters JM, Moore GT. Attributes of child care centers and outdoor play areas associated with preschoolers' physical activity and sedentary behavior. Environ Behav. 2012;44(3):334-49. https:// doi.org/10.1177/0013916510393276.

82. Tandon PS, Saelens BE, Christakis DA. Active play opportunities at child care. Pediatrics. 2015;135(6):e1425-31. https://doi.org/10.1542/peds.2014-2750.

83. Vale S, Santos R, Silva P, Soares-Miranda L, Mota J. Relationship of objective measurement of physical activity during school hours and BMI in preschool children. Int J Pediatr Obes. 2011;6(2):37-8l. https://doi.org/10.3109/ 17477166.2011 .613663$.

84. Vale S, Santos R, Silva P, Soares-Miranda L, Mota J. Preschool children physical activity measurement: Importance of epoch length choice. Pediatr Exerc Sci. 2009;21(4):413-20. https://doi.org/10.1123/pes.21.4.413.

85. Van Cauwenberghe E, De Bourdeaudhuij I, Maes L, Cardon G. Efficacy and feasibility of lowering playground density to promote physical activity and to discourage sedentary time during recess at preschool: a pilot study. Prev Med. 2012;55(4):319-21. https://doi.org/10.1016/.ypmed.2012.07.014.

86. Van Cauwenberghe E, De Craemer M, De Decker E, De Bourdeaudhuij I, Cardon G. The impact of a teacher-led structured physical activity session on preschoolers' sedentary and physical activity levels. J Sci Med Sport. 2013;16(5):422-6. https://doi.org/10.1016/j.jsams.2012.11.883.

87. Williams HG, Pfeiffer KA, O'Neill JR, Dowda M, Mclver KL, Brown WH, et al. Motor skill performance and physical activity in preschool children. Obesity. 2008;16(6):1421-6. https://doi.org/10.1038/oby.2008.214.

Ready to submit your research? Choose BMC and benefit from:

- fast, convenient online submission

- thorough peer review by experienced researchers in your field

- rapid publication on acceptance

- support for research data, including large and complex data types

- gold Open Access which fosters wider collaboration and increased citations

- maximum visibility for your research: over $100 \mathrm{M}$ website views per year

At $\mathrm{BMC}$, research is always in progress.

Learn more biomedcentral.com/submission 